ACTA UNIVERSITATIS LODZIENSIS

Folia Litteraria Romanica 16, 2021

https://doi.org/10.18778/1505-9065.16.23

Łukasz Szkopiński

Université de Łódź

(iD https://orcid.org/0000-0002-0486-600X

lukasz.szkopinski@uni.lodz.pl

\title{
Une peinture sombre de l'amour dans Les Ombres sanglantes
}

\begin{abstract}
RÉSUMÉ
La présente étude a pour objectif de se pencher sur le thème de l'amour dans Les Ombres sanglantes (1820) de J. P. R. Cuisin. Quatre histoires de ce recueil seront analysées dans ce but : La demeure d'un parricide, ou le triomphe du remords; L'Infanticide, ou la fausse vertu démasquée; La guérite de la religieuse, ou la vestale prévaricatrice; et Niobé, ou l'élève de la nature. On s'intéressera particulièrement aux crimes décrits par l'auteur dans chacun de ces récits : le parricide, l'infanticide, la rupture des vœux religieux et l'inceste. La manière violente, corrosive et parfois caricaturale dont les différentes relations sentimentales, souvent de nature illicite, voire pathologique, sont peintes dans Les Ombres sanglantes diverge considérablement de la vision de l'amour typique du roman noir tel qu'il se pratique en France à la fin du XVIII ${ }^{\mathrm{e}}$ siècle et au début du XIX', et marque clairement l'avènement du genre frénétique de plus en plus présent dans la littérature française de cette période.
\end{abstract}

MOTS-CLÉS - amour, littérature française, roman frénétique, J. P. R. Cuisin, Les Ombres sanglantes

\section{A Dark Portrayal of Love in Les Ombres sanglantes}

\section{SUMMARY}

The aim of this paper is to explore the theme of love in Les Ombres sanglantes [The Bloody Shadows] (1820) by J. P. R. Cuisin. Four stories from the volume were chosen for this purpose, including "La demeure d'un parricide, ou le triomphe du remords"; "L'Infanticide, ou la fausse vertu démasquée"; "La guérite de la religieuse, ou la vestale prévaricatrice" and "Niobé, ou l'élève de la nature". The analysis of these texts revolves around the crimes described by the author in each of the stories, namely parricide, infanticide, breach of religious vows and incest. The violent, destructive and sometimes grotesque manner in which these various sentimental relationships, often of an illicit or even pathological nature, are portrayed in Les Ombres sanglantes diverges considerably from the vision of love typical for the Gothic novel in France at the end of the $18^{\text {th }}$ and the beginning of the $19^{\text {th }}$ centuries, clearly marking the advent of the frenetic genre, more and more present in the French literature of this period.

KEYWORDS - love, French literature, frenetic novel, J. P. R. Cuisin, Les Ombres sanglantes 
J. P. R. Cuisin fut un auteur prolifique, bien que ses œuvres soient pour la plupart restées anonymes. C'est probablement pour cette raison que Pigoreau, dans sa Bibliographie biographico-romancière (1821), observe : " sur la parole de nos confrères, éditeurs de ses ouvrages, nous avons mis sous son nom plusieurs écrits, pour lesquels nous sommes prêts à nous rétracter, s'il les désavoue »(Pigoreau, 1821 : 174). La longue liste de ses ouvrages reste en contraste avec le peu que nous savons sur sa vie. Quant à ses prénoms, nous n'en connaissons que les initiales et même cela suscite quelques controverses, vu que différentes sources proposent des variantes assez diverses : le plus souvent « J. P. R. », mais aussi « J. P. », « J. R. P. » ou uniquement «P. ». Il est né en 1777 , mais la date de sa mort est un nouveau mystère : il serait « mort gardemagasin de poudres vers $1845 »$ (Quérard, 1854 : 119). Un court article qui lui a été consacré en 1883 dans la revue Le Livre confirme un manque presque total d'informations sur Cuisin : « aucun biographe n'a, que nous sachions, pris la peine de consacrer le plus petit article à cet écrivain qu'il serait plus juste d'appeler un écrivassier ». Bien que l'auteur du texte considère Cuisin comme « un des compilateurs les plus féconds de la première moitié de ce siècle » (Le Livre, 1883 : 793), il le qualifie aussi d' « écrivain famélique » et de «pauvre écrivain », en ajoutant, avec le dédain typique d'un critique de la fin du XIX siècle pour la littérature populaire du début de ce même siècle, que « sur cent volumes et plus qu'il a publiés, [il] n'en a pas laissé un seul ayant une véritable valeur littéraire, ou du moins une utilité quelconque » (Le Livre, 1883 : 794). Enfin, il nous reste à citer une note consacrée à Cuisin dans le Dictionnaire des gens de lettres vivants (1826). Selon la page de titre de cet ouvrage facétieux, il fut publié « par un descendant de Rivarol », mais plusieurs sources, comme le catalogue de la Bibliothèque nationale de France, lèvent son anonymat : il s'agirait en fait de Cuisin lui-même, aidé par un certain G.-L. Brismontier. Il y est décrit comme « un véritable modèle de versatilité » à qui on ne peut "refuser quelque esprit, quelque chaleur d'imagination » même si « la sagesse n'en règle jamais les bonds, les soubres-sauts et les saccades ». Quant à « ses principaux trophées », ce sont " des in-18 d'un style parfois érotique : avec des mœurs pures, de la probité, il a allié ce contraste adultère ». Si le texte est vraiment écrit, ou au moins approuvé, par Cuisin, il faut souligner son caractère auto-ironique particulièrement visible dans le passage suivant : "Malgré tous ces efforts, il ne parviendra jamais à faire peau neuve, et la postérité ne prononcera jamais le mot de camelotte, sans qu'une réminiscence ne le signale comme un de ses coryphées les plus chauds » ([Brismontier, Cuisin,] $1826: 78-79)$.

Les Ombres sanglantes, Galerie funèbre de prodiges, Événements merveilleux, Apparitions nocturnes, Songes épouvantables, Délits mystérieux, Phénomènes terribles, Forfaits historiques, Cadavres mobiles, Têtes ensanglantées et animées, Vengeances atroces, et combinaisons du crime puisés dans des sources réelles. Recueil propre à causer les fortes émotions de la terreur (1820), pour l'évoquer 
sous son titre complet, constitue sans doute l'un des ouvrages les plus connus de Cuisin et, contrairement aux déclarations de l'éditeur de la revue citée cidessus à propos de la qualité des écrits de Cuisin, nous semble être un ouvrage fort intéressant. De nombreuses références que l'auteur du recueil fait à la littérature, aussi bien classique que contemporaine, ainsi que ses réflexions sur les goûts littéraires de l'époque, exposées avec beaucoup d'humour et avec pas mal d'ingéniosité dans l'introduction de l'ouvrage et continuées par la suite dans Les Fantômes nocturnes (1821), prouvent que Cuisin était beaucoup plus qu'un « écrivassier ». Considéré par certains comme une simple parodie littéraire, le texte échappe pourtant aux catégories et aux jugements univoques.

$\mathrm{Au}$ moment de la publication du recueil, le modèle traditionnel du roman gothique (à la Radcliffe), très à la mode en France à partir de la fin du XVIII ${ }^{e}$ siècle, quoique toujours en vogue sous la Restauration, devient de plus en plus anachronique et, sous les influences romantiques, il évolue et prend des formes beaucoup plus diverses. C'est le cas de "l'école frénétique ", pour utiliser l'expression de Charles Nodier (1821:83), qui gagne clairement en popularité à cette époque-là. Une analyse détaillée de ce sujet ou la comparaison entre le roman frénétique naissant et le roman gothique classique, pour ainsi dire, dépasseraient largement le cadre de cette modeste étude ${ }^{1}$. Cependant, il faut observer que malgré de nombreuses filiations entre les deux genres, il existe aussi beaucoup de différences parfois fondamentales. À titre d'exemple, tandis que « le gothique est une littérature du réconfort » (Seth, 2010 : 36), dans la littérature frénétique « le mal est effectif, et la peur, qui découle de l'attente d'un événement violent, est remplacée par l'horreur, par la confrontation brutale avec l'événement redouté » (Pézard, 2013 : 46-47).

Dans la présente étude, nous questionnerons la place de l'amour dans Les Ombres sanglantes. Pour ce faire, nous présenterons quatre histoires issues de ce recueil, dans lesquelles ce sentiment joue un rôle particulièrement important dans la trame narrative. Nous divisons notre article en sections dont les titres renvoient aux crimes commis par les protagonistes et motivés par diverses formes d'amour et de désir, parfois nettement pathologiques. Nous conclurons notre analyse par quelques réflexions sur la nature frénétique de l'ouvrage.

\section{Parricide ou un choix épouvantable}

La demeure d'un parricide, ou le triomphe du remords, qui ouvre le recueil, raconte l'histoire d'Amédée, dont le père, le vieux baron d'Altamongues, veut qu'il épouse Christine de Melsinberg, la fille d'un ami qui lui a sauvé la vie pendant la guerre. Cependant, le jeune homme tombe amoureux de Blanche de

${ }^{1}$ Cf. A. Glinoer (2009), La littérature frénétique, Paris, Presses Universitaires de France. 
Lindorff, qui s'avère la fille de l'ennemi mortel du vieux baron de sorte que ce dernier refuse absolument de consentir à ce mariage. Finalement, pour éviter les noces forcées, Amédée décide de tuer son père, mais ses remords et la vengeance de Christine concourent à sa perte.

Rien ne présage la tragédie à venir quand le narrateur nous décrit la première rencontre entre les amants et le sentiment qui commence à s'épanouir entre eux. « La voir, l'admirer dans sa danse légère et noble, toucher de sa main, tremblante des feux soudains d'un premier amour, sa main d'albâtre, et respirer dans ses beaux yeux noirs la passion la plus violente, fut pour Amédée l'effet de l'éclair » $(\mathrm{I}, 35)^{2}$. Par la suite, la flamme amoureuse qui lie Blanche et Amédée est comparée par Cuisin à « une chaîne comme électrique » qui « avait uni tacitement les deux amans les plus enivrés d'une égale ardeur » (I, 36).

Cependant, le lecteur est vite confronté à un brusque changement de genre : ce qui semblait un roman sentimental se transforme tout à coup en un ouvrage frénétique. Ce changement affecte également la manière dont les caractères des personnages sont décrits. Le protagoniste, initialement présenté au lecteur comme " ce cœur sensible, né pour la vertu » (I, 34), devient « le monstre Amédée » (I, 62), tandis que le baron, d'abord dépeint comme « un despote insensé, esclave d'un vain engagement, ou plutôt de son orgueil » (I, 51) est désormais perçu comme la victime d'un crime révoltant.

Finalement, malgré son caractère premier, celui d'un amour pur et des plus romantiques, la relation entre Amédée et Blanche conduit à un parricide. Le narrateur souligne à plusieurs reprises la nature destructrice de ce sentiment, notamment à travers le constat que « c'est donc le sang d'un père qui va expier les chagrins de l'amour...» (I, 53), ou lorsqu'il conclut que « [...] dans ces scènes sanglantes, l'amour, foyer trop commun des plus terribles passions, ne marchait plus qu'à la lueur des torches de l'envie et de la vengeance » $(\mathrm{I}, 75)$.

Il vaut la peine de noter le rôle de Christine de Melsinberg, la fiancée rejetée par le protagoniste, dans la résolution du mystère entourant la mort du baron. Bien qu'Amédée ait tenté de masquer son crime afin d'en faire porter la faute à une troupe de brigands, peu à peu, Christine « soupçonna la vérité, et seulement éclairée des lumières de sa jalousie, entreprit tout pour la découvrir » (I, 71). Le dénouement du récit ainsi conduit peut donner l'impression d'un monde à l'envers : d'un côté, l'amour pousse le fils à tuer son père; de l'autre, la haine, accompagnée du désir de vengeance, semble mener à la vérité et à la punition du coupable. L'élément commun de ces deux passions, a priori nettement antagonistes, c'est que leur force effrénée annihile, d'une manière ou autre, tous les personnages principaux de l'histoire.

2 Toutes les citations de l'ouvrage proviennent de l'édition suivante : J. P. R. Cuisin, Les Ombres sanglantes, Paris, Lepetit, 1820. Le volume et la page sont indiqués entre parenthèses après chaque extrait. Nous avons conservé la graphie originelle des passages cités. 


\section{Infanticide : d'un drame à l'autre}

Clotilde, protagoniste de L'Infanticide, ou la fausse vertu démasquée, est bien connue des cercles parisiens : sa mère, Madame Dorlange, reçoit en effet dans sa maison du Faubourg Saint-Germain « tout ce que la bonne société a de plus distingué dans les deux sexes » (I, 228). Un jour, la jeune fille rencontre le colonel Merville. Une relation galante se noue entre eux et Clotilde se laisse séduire par le militaire. L'idylle sentimentale se termine quand elle se rend compte de sa grossesse.

La première partie de l'histoire correspond assez peu au titre apparemment choisi pour faire sensation. Le lecteur est d'abord témoin du jeu que mènent Clotilde et son nouvel admirateur, combinant convenances sociales et manifestations du désir. Mademoiselle Dorlange cherche « un terme conciliateur qui puisse conserver à la fois, ainsi que le dit si spirituellement la marquise de Merteuil, et les plaisirs du vice, et les honneurs de la vertu » (I, 235). Cette allusion aux Liaisons dangereuses semble pertinente lorsqu'on considère les efforts de Merville qui, en nouveau Valmont, fait de son mieux pour achever sa conquête. La métaphore faisant de l'amour une guerre entre les amants est d'ailleurs très claire dans plusieurs passages, notamment quand il nous est dit qu'autant le colonel « avait été rusé militaire, prompt à faire jouer sur son ennemi des mines, des manœuvres perfides, autant il savait attaquer le beau-sexe avec art, et surprendre, d'un brillant coup de main, les sentinelles les plus assidues de la modestie, de la vigilance et de la pudeur » (I, 230). Dans un autre extrait, cet « ancien colonel d'hussards, amant et amant aimé [...] se dit qu'une surprise, une charge brillante, à la guerre comme en amour, est le grand moyen des conquêtes » (I, 237). Finalement, la protagoniste cède à " son aimable vainqueur » $(\mathrm{I}, 251)$ et le couple passe ensemble une nuit de passion. Décrivant par la suite les tendres sentiments des deux amants, quand ils se réveillent le lendemain l'un près de l'autre, le narrateur se livre même à une série de réflexions sur l'amour sensuel ${ }^{3}$.

Or ici, tout comme dans la première histoire, la tonalité du récit change brusquement. D'un ouvrage libertin, le lecteur se trouve soudainement transporté au milieu d'un roman frénétique. Quand Clotilde se rend compte de sa grossesse, elle veut l'interrompre, mais, à la suite du refus formel de son amant de lui venir en aide, la jeune fille donne secrètement le jour à un garçon. Peu après, elle prend

\footnotetext{
3 « Le petit jour vint les surprendre dans le plus voluptueux abandon. La belle Clotilde, le front couronné d'un bras, le sein tout-à-fait nu, sommeillait encore, accablée d'une fatigue délicieuse. Merville lui avait juré tant de fois qu'il serait discret et fidèle !... Ce fut son amant qui se réveilla le premier. Est-il, en situation d'amour, un moment plus délectable que celui d'un lendemain ? La passion, l'amour-propre, la gloire d'avoir triomphé d'une vertu rebelle, de contempler, de parcourir sans aucun voile importun des attraits qui, jusqu'alors, avaient été toujours scrupuleusement dérobés à votre vue... de porter ses lèvres sur des trésors animés, de faire mille envieux... Non, l'homme dans cette situation céleste ne paierait trop ces extases d'un demi-siècle d'adversité !... » (I, 248-249).
} 
la fatale résolution de tuer son fils, en l'enterrant vif dans le jardin. Le narrateur ne nous épargne aucun détail :

[...] elle précipite une dernière fois dans ce cercueil creusé de ses propres mains la malheureuse créature ; et sans entrailles, sans miséricorde pour les derniers cris qu'elle fait entendre, Clotilde a la barbarie de les étouffer tout-à-fait, ces cris, en emplissant des premières pelletées de terre cette bouche innocente qui suffoque, et expire en balbutiant les derniers accens de sa débile douleur... (I, 256).

Étant donné que, dans le recueil de Cuisin, tout crime doit inévitablement être suivi d'un châtiment, Clotilde, sous le poids des remords et terrifiée par la punition qui l'attend, se donne la mort. À cette occasion, le narrateur donne de nouveau libre cours à sa veine macabre, en peignant une scène d'une violence forcenée : « ses muscles, ses nerfs, ses veines gonflées se brisent comme des ressorts d'acier dans une trop grande tension; et son sein meurtri, arraché de ses propres mains, n'offre plus que des lambeaux de chair informes, souillés d'un mélange odieux de lait et de sang... » (I, 258-259).

Il est encore une autre similitude entre le présent récit et le précédent vu que, dans les deux cas, le changement de tonalité a des répercussions sensibles sur la façon de décrire le caractère de certains personnages. C'est particulièrement visible pour Merville. Le colonel, jusqu'alors présenté comme un libertin insouciant, devient tout à coup un modèle de vertu : lorsque Clotilde lui demande « un breuvage avortif», il « frémit d'horreur ». Sans doute conscient de cette légère incohérence dans la personnalité du militaire, l'auteur se voit obligé d'apporter, par le truchement du narrateur, l'explication suivante :

Il veut bien conquérir par ruse les attraits vierges d'une jeune beauté, et son honneur de colonel de hussards n'en reçoit pas la plus petite atteinte ; mais devenir le complice d'un infanticide, porter un poison destructeur dans le sein d'une innocente créature, attaquer par des potions homicides la santé de la mère et de l'enfant !... cette seule idée l'épouvante et il ne peut se défendre d'une secrète haine, d'un certain mépris pour celle qui a pu lui faire de semblables propositions... (I, 252-253).

\section{Rupture des vœux religieux : entre l'amour de Dieu et la passion}

Palmira Monte Hermoso, l'héroïne de la nouvelle La guérite de la religieuse, ou la vestale prévaricatrice, après avoir été séparée de son bien-aimé Fernando, refuse tous les autres partis que lui proposent ses parents, et se voit par conséquent obligée de prendre le voile. Toutefois, un jour, Fernando parvient à la retrouver et, malgré les vœux prononcés par la jeune fille et une série d'événements surnaturels, ils redeviennent amants. Cette histoire, comme la majeure partie des récits présentés dans l'ouvrage, se termine de manière tragique : Palmira, enceinte, est tuée alors qu'elle tente de s'enfuir du couvent. 
Cuisin explore ici un motif littéraire de plus en plus usité tout au long de la seconde moitié du XVIII siècle ${ }^{4}$ et au-delà : celui des vœux forcés. Cependant, bien qu'ayant informé le lecteur que la protagoniste « avait été jetée de force dans le couvent de la Penitencia » et qu'elle « avait été contrainte, par les plus cruelles violences, de prendre l'habit et de prononcer ses vœux » (II, 181), le narrateur témoigne relativement peu de compassion à cette dernière, et fait encore moins preuve de compréhension concernant sa relation interdite avec Fernando, « cet amant sacrilège », estimant que « la fougue aveugle des passions lui fait franchir en un instant les bornes sacrées de l'honneur et de la religion » (II, 193).

L'implacabilité du narrateur répond parfaitement au but de l'ouvrage, qui vise à choquer le public en lui donnant à voir un vaste échantillon de vicissitudes humaines. Logiquement, au lieu de s'apitoyer sur le sort tragique des amants, Cuisin se concentre sur l'aspect sacrilège de leur relation.

Afin de mieux accentuer la nature coupable de cet amour et de mettre en valeur la punition céleste qu'il déclenche, l'auteur se sert d'un autre moyen d'importance : le surnaturel. Ainsi donc, la première fois que Fernando voulut parvenir à la cellule de Palmira, " un fantôme chargé de draperies couvertes de larmes de sang, tenant dans la main un lis et une croix, symboles de la pureté et de la religion, vint l'arrêter de son aspect effroyable » (II, 195-196). À une autre occasion, " au moment où il veut se précipiter dans ses bras, L'OMBRE SANGLANTE se place entre elle et lui, et, d'un air formidable, leur oppose le lis et la croix » (II, 198-199). Même le dénouement tragique de cette histoire ne met pas fin aux apparitions mystérieuses liées au couvent de la Penitencia de Salamanque. En effet, pendant longtemps, le jour de l'anniversaire de la mort de Palmira, les habitants voient « des monstres ailés investir la fatale guérite, en remplissant l'air de ce cri effrayant : MORT AU PARJURE !... » Le narrateur précise que « ces génies infernaux [...] ne disparaissaient qu'au lever de l'aurore, en laissant dans l'air une odeur affreuse de soufre » (II, 206).

\section{Inceste ou une expérience diabolique}

Dans Niobé, ou l'élève de la nature. Mœurs parisiennes, on découvre l'histoire de St.-Hilaire, qui fait croire à la mort de sa fille nouveau-née afin de la cacher dans les souterrains de sa maison secrète, de la convertir en une sorte d'enfant sauvage et de lui faire subir, pendant des années, une expérience révoltante de nature sociale et sexuelle.

$\mathrm{Au}$ début du texte, le chevalier de St.-Hilaire est présenté comme un bon vivant, un « brillant libertin de la Chaussée-d'Antin ». Quand il décide enfin de

${ }^{4}$ Cf. M. Choudhury, Convents and Nuns in Eighteenth-century French Politics and Culture, Cornell University Press, 2004. 
se marier, il ne le fait « que par ton, par lassitude de la vie de célibataire ». Le narrateur ajoute que cet homme singulier « n'avait pris femme que comme on ferait l'acquisition d'un équipage d'une piquante invention, et seulement pour jouir des plaisirs sans conséquence d'une simple nouveauté » (I, 131-132). Finalement, en expliquant pourquoi « il se plaça vivant dans le cercueil conjugal », il constate : « Il faut y tâter un peu de tout ; je veux voir ce que c'est que ça » (I, 132).

Ce ton léger et facétieux disparaît soudainement, quand le narrateur décrit en détail le projet incestueux du chevalier. Cette entreprise est d'autant plus épouvantable qu'elle est préméditée, fait souligné à maintes reprises dans le récit. Il s'agit bien là, de la part de St.-Hilaire, « d'une froide analyse » $(I, 136)$ dont procède la mise en œuvre d'une " pièce d'expérience de ses monstrueuses hypothèses » $(\mathrm{I}, 138)$, qui franchit « les bornes les plus sacrées de la nature et de la morale » (I, 137).

Le temps passant, l'accent est mis toujours davantage sur l'aspect sexuel de la triste existence de Niobé dans la correspondance entre St.-Hilaire et Florimont, son complice et seul confident. Bien que l'on relève dans l'ouvrage de nombreuses scènes d'une violence inouïe, souvent très explicites, Cuisin n'en use pas aussi librement avec des descriptions potentiellement pornographiques, qui manqueraient sans doute aux bienséances. Aussi le narrateur déclare-t-il que les détails des missives des deux libertins sont « trop scandaleux pour souffrir le jour de l'impression », et décide-t-il de les supprimer " pour n'en faire connaître en substance que les faits qui forment le dénouement de cette histoire » (I, 161).

C'est justement dans une lettre à Florimont que St.-Hilaire raconte le moment qu'il a attendu pendant les seize premières années de son « expérience », celui de son premier contact sexuel avec Niobé :

Je suis enfin monté sur ce trône d'ivoire et d'ébène que je brûlais d'usurper. Cette charmante Niobé, depuis seize mortelles années, captive pour mon bonheur, a vu tomber en un instant ses chaînes avec la fleur de sa virginité.... Enfin, je la rends au monde, et elle y entre par la porte du plaisir. Couronnée des plus belles roses virginales que l'on puisse cueillir dans le jardin d'Italie, un charmant bouton de rose s'est épanoui sous mes baisers brûlans... Quelle félicité ! La scène vit encore dans mon âme, et ma plume ne peut exprimer les délices de ce voluptueux trépas (I, 155-156).

Il est intéressant de noter dans ce passage le net contraste entre la manière élégante et poétique dont cet événement est décrit, et la réalité choquante et criminelle à laquelle il renvoie.

À la fin de l'histoire, la vérité est découverte. Niobé est enfin libre tandis que son père doit faire face à la justice. Les malheurs de cette " élève de la nature » (I, 158) ne se terminent pas là pour autant : confrontée à « cette célébrité scandaleuse » (I, 169) et incapable de supporter la curiosité malsaine de la société parisienne, mue par le goût du spectacle et la quête de sensations fortes, Niobé fuit le monde en prenant le voile. 
En lisant Les Ombres sanglantes, on trouve partout des éléments typiques du roman noir de la fin du XVIII siècle et du début du XIX ${ }^{\mathrm{e}}$. Le décor auquel recourt Cuisin dans plusieurs de ses histoires en constitue une excellente illustration : des châteaux médiévaux, une forêt sombre, des souterrains, les forces déchaînées de la nature telles que les représente, par exemple, la description de l'" ouragan affreux » (I, 46) qui accompagne les entreprises meurtières d'Amédée, etc.

Cela étant dit, l'emploi excessif de ces ingrédients stéréotypés du genre amène vite le lecteur à nourrir certains doutes quant au véritable caractère de l'ouvrage. Alice Killen observe notamment que «Cuisin, qui parodie ce genre "noir" dans ses Ombres sanglantes et ses Fantômes nocturnes, y résume tout ce qu' il y a d'effrayant dans le roman de cette époque » (Killen, 2000 : 168). Cette remarque n'est pas dépourvue de vérité, surtout si l'on prend en considération le ton ludique de l'introduction ainsi que le titre baroque du recueil. Or la question s'avère beaucoup plus complexe, l'ouvrage de Cuisin présentant également de nombreux traits du roman frénétique naissant : la fascination du mal, la multiplication de scènes et de descriptions violentes, l'accent porté sur le personnage du bourreau plutôt que sur ses victimes, l'emploi du surnaturel, etc. L'image de l'amour développée par l'auteur se conforme aussi aux exigences de ce genre particulier. Dans les romans noirs à la Radcliffe, la trame sentimentale joue un rôle fondamental. Certes, les protagonistes doivent faire face aux méchancetés des scélérats qui les persécutent, mais en général ce conflit reste étroitement lié à leur quête amoureuse. À la fin, presque invariablement, le mal est puni, laissant place au triomphe de la vertu, renforcé par l'image du bonheur des amants qui, après d'innombrables péripéties, peuvent enfin passer ensemble le reste de leur vie. Tel n'est plus le cas dans les ouvrages de Cuisin et d'autres écrivains frénétiques, dont le but premier n'est plus de prêcher les valeurs morales, mais de conjuguer « les grandes secousses [et] les grandes émotions de l'âme ", comme l'affirme Cuisin dans un recueil ultérieur (Les Fantômes nocturnes, 1821 : I, 4). L'amour lui-même est soumis à cet objectif, ce que nous percevons clairement dans l'ouvrage analysé.

Maurice Lévy, en analysant l'œuvre gothique d'Ann Radcliffe et celle de Matthew Gregory Lewis, souligne une nette opposition entre la terreur et l'horreur, chacune de ces notions distinguant l'un de ces deux écrivains. Il observe que " la terreur se nourrissait de doutes » alors que " l'horreur s'installe dans une abominable certitude », et constate : " [1'horreur] n'est plus imagination, mais vision. Elle se réfère plus au possible, elle est au cœur du réel » (Lévy, 1995 : 341-342). Nous sommes d'accord avec Émilie Pézard quand elle remarque que « cette opposition permet de comprendre la spécificité du romantisme frénétique » (Pézard, 2017). C'est précisément en suscitant un sentiment d'horreur chez ses lecteurs que Cuisin entend produire ces « grandes secousses » et ces " grandes émotions de l'âme », déjà mentionnées ci-dessus, mais aussi répandre « un 
précieux effroi dans l'âme des êtres dépravés » (1821: I, 14). Peu importent alors, dans les histoires évoquées au sein de cette étude, la manière peu cohérente de construire les personnages, l'omniprésence de scènes violentes et révoltantes, frôlant parfois le ridicule, et la grande hybridité stylistique que nous avons mises en évidence, du moment qu'elles contribuent à surprendre le lecteur, voire à le choquer, par le contenu auquel il s'attend le moins.

Dans son livre La littérature frénétique, Anthony Glinoer évoque « des recueils de contes qui semblent toujours osciller entre le répertoire d'histoires à lire pour le plaisir de se faire peur et la parodie » (Glinoer, 2009 : 72). L'on peut ainsi affirmer, en guise de conclusion, que Les Ombres sanglantes de Cuisin illustrent à merveille cette définition.

\section{Bibliographie}

[BRISMONTIER, G.-L., CUISIN, J. P. R.] (1826), Dictionnaire des gens de lettres vivants, Paris

CHOUDHURY, Mita (2004), Convents and Nuns in Eighteenth-century French Politics and Culture, Cornell University Press

CUISIN, J. P. R. (1820), Les Ombres sanglantes, Paris, Lepetit

CUISIN, J. P. R. (1821), Les Fantômes nocturnes, Paris, Lepetit

GLINOER, Anthony (2009), La littérature frénétique, collection « Les Littéraires », Paris, Presses Universitaires de France

KILLEN, Alice (2000 [1915/1967]), Le Roman terrifiant ou roman noir de Walpole à Anne Radcliffe et son influence sur la littérature française jusqu'en 1884, Genève, Slatkine Reprints

Le Livre. Bibliographie Moderne, quatrième année, le 10 décembre 1883

LÉVY, Maurice (1995), Le roman " gothique » anglais 1764-1824, Paris, Albin Michel

NODIER, Charles (1821), « Le Petit Pierre, traduit de l'allemand, de [Christian Heinrich] Spiess », Annales de la littérature et des arts, t. 2, Paris, p. 77-83

PÉZARD, Émilie (2013), " La vogue romantique de l'horreur : roman noir et genre frénétique », Romantisme, $\mathrm{n}^{\circ} 160$ (2), p. 41-51

PÉZARD, Émilie, «Un genre fondé sur le « goût de l'atroce ». Le romantisme frénétique », Fabula / Les colloques, Les genres littéraires, les genres cinématographiques et leurs émotions (http:// www.fabula.org/colloques/document4094.php, consulté le 13 février 2020)

PIGOREAU, Nicolas-Alexandre (1821), Bibliographie biographico-romancière, ou Dictionnaire des romanciers, Paris, Pigoreau

QUÉRARD, Joseph-Marie (1854), Les Écrivains pseudonymes et autres mystificateurs de la littérature française, t. 11, Paris

SETH, Catriona (2010), Imaginaires gothiques. Aux sources du roman noir français, Paris, Desjonquères

Lukasz Szkopiński - maître de conférences à l'Institut d'Études Romanes de l'Université de Łódź. Il est auteur de L'Euvre romanesque de François Guillaume Ducray-Duminil (Classiques Garnier, 2015), de l'édition critique de Victor, ou l'Enfant de la forêt (1797) de F. G. DucrayDuminil (Classiques Garnier, 2019) et de nombreux articles concernant le roman noir et la littérature française de la fin du XVIII ${ }^{e}$ siècle et du début du XIX ${ }^{\mathrm{e}}$. Łukasz Szkopiński est directeur de la revue scientifique e-Scripta Romanica. 